\title{
Factor proportionality in multiple households closed CGE models: theory and illustrations
}

\author{
Yves Balasko • Octavio Tourinho
}

Received: 11 December 2013 / Accepted: 10 March 2014 / Published online: 28 March 2014 (C) SAET 2014

\begin{abstract}
The factor contents of different consumers' consumption bundles computed from a country's CGE model with multiple households are seen to be proportional or almost proportional in the case of four different countries, namely USA, Brazil, Spain and Ivory Coast. These countries have been chosen because of the existence for these countries of CGE models with multiple households (by opposition to a representative consumer) and good quality data. This study has been limited to these four countries because we thought that the implications for CGE modeling of our findings should not delay any further their publication even though further studies are obviously needed. Taken as a law, we show that proportionality of factor contents at equilibrium for all endowment vectors and fixed preferences implies that households' implicit preferences for factor contents are identical and homothetic. Another consequence is that factor and goods' equilibrium prices are unique and depend only on total factor resources, not on the distribution of these factors between households. Factor proportionality may therefore be at the root of the puzzling rigidity and uniqueness of equilibrium prices of goods and factors observed in CGE models with multiple households.
\end{abstract}

Electronic supplementary material The online version of this article (doi:10.1007/s40505-014-0032-x) contains supplementary material, which is available to authorized users.

The authors of this paper are grateful to J. Hutton, T. Kehoe, N. Kiefer, T. Mitra, K. Shell, M. Tvede and two anonymous referees for their thoughtful comments and suggestions. The research reported in this paper would have been impossible without the warm hospitality and generous financial support of the SPP fund at the Department of Economics and Related Studies (University of York), of the Seedcorn fund also of the University of York and, in Rio de Janeiro, of UERJ, IMPA and PUC-Rio de Janeiro.

\footnotetext{
Y. Balasko $(\varangle)$

Department of Economics and Related Studies, University of York, York, UK

e-mail: yves@balasko.com

O. Tourinho

Department of Economics, UERJ, Rio de Janeiro, Brazil
} 
Keywords Factor proportionality · Price rigidity · Equilibrium uniqueness CGE models

JEL Classification $\quad$ C67 $\cdot$ C68 $\cdot$ D51 $\cdot$ D58

\section{Introduction}

The factor contents of different consumers' consumption bundles computed from a country's CGE model with multiple households are seen to be proportional or almost proportional in the case of four different countries, namely USA, Brazil, Spain and Ivory Coast. These countries have been chosen because of the existence for these countries of CGE models with multiple households (by opposition to a representative consumer) and good quality data. This study has been limited to these four countries because we thought that the implications for CGE modeling of our findings should not delay any further their publication, though further studies are obviously needed. The proportionality in factor contents in multiple households CGE models is likely to be the result of more or less implicit and seemingly innocuous simplifying assumptions made at various stages of the modeling process. Taken as a law, we show that factor proportionality is not innocuous at all. We start by showing that multiple households closed CGE models (i.e., without foreign and governmental sectors) are equivalent to pure exchange models where households (also known as the consumers in those models) have preferences for factors induced by their preferences for consumption goods in the CGE models. We then apply this equivalence to show that in exchange economies, factor proportionality for all endowment vectors for given preferences is equivalent to those preferences being identical and homothetic. Applied to our closed CGE models, households' implicit preferences for factor contents induced by their preferences for consumption goods are therefore identical and homothetic. This identity of preferences for factors does not require that households' preferences for consumption goods are identical and, in general, they are not in the multiple households CGE models that we have studied. Another consequence of factor proportionality for all endowment vectors is that factor and goods' equilibrium prices are unique and depend only on total factor resources, not on the distribution of these factors between the households of a given country in the case of CGE models. Factor proportionality offers one a possible explanation of the mysterious rigidity and uniqueness of equilibrium prices of goods and factors observed in many CGE models. If factor proportionality is confirmed to be a property of the current versions of multiple households CGE models, further research will be needed to identify the implicit assumptions in CGE modeling at the root of that factor proportionality, assumptions that will have to be eliminated if one wants to get more relevant CGE models with multiple households.

Computable general equilibrium or CGE models are numerical implementations of the general equilibrium models that are based on real world data. A huge literature has been devoted to the study of the general equilibrium model since its formulation by Walras (1874). The existence of equilibrium (Arrow and Debreu 1954; McKenzie 1954) and the efficiency of equilibrium allocations (Arrow 1951a) are the best known properties of that model. Just after them, the multiplicity of equilibria stands out for its 
combination of theoretical interest with importance in applications. This explains the search for economically relevant conditions that imply the uniqueness and stability of equilibrium. First results were obtained by Wald (1951). The search was pursued by Arrow, Hahn, Hurwicz, McKenzie and Uzawa among many others during the 1950s and 1960s. See the survey article by Negishi (1962) and the two chapters on uniqueness and tatonnement stability in Arrow and Hahn (1971) monumental book for an account of the results of that period. A new line of research was started in the wake of Debreu's theory of regular economies (Debreu 1970) by Dierker (1972) and Kehoe $(1980,1982)$ who proved index theorems for exchange and production economies. Important by-products of those index theorems were more general sufficient conditions for the uniqueness of equilibrium in general equilibrium models, conditions that have been readily applied by Kehoe and Whalley $(1985)$ and Kehoe $(1985,1991)$ to CGE models. Kehoe's example of a production economy with multiple equilibria (Kehoe 1984) shows, however, that uniqueness is not satisfied in very general classes of general equilibrium models.

In the less complex exchange model, it is very easy to create examples of economies with multiple equilibria provided preferences are not homothetic and identical. It suffices to select in any given fiber of the equilibrium manifold (Balasko 1975a) an equilibrium with a negative index number. In general, this requires little more than a large net trade vector. Then it follows from the index theorem for exchange economies that an economy that features an equilibrium with a negative index number has other equilibria. (See Balasko (2011) for more details.) All these results imply the existence of economies with multiple equilibria for some endowment vectors at least as a consequence of the non-linearities of the general forms of general equilibrium model.

The absence of multiple equilibria in the many CGE models has been noticed by sufficiently many economists to become a phenomenon in need of an explanation given the widespread occurrence of multiple equilibria in general equilibrium models of exchange and production economies. This uniqueness of equilibrium is in fact a real problem only for CGE models with multiple households because equilibrium is obviously unique as soon as there is a representative household or, equivalently, a unique consumer. The rigorous study of the question of the number of equilibria in CGE models with multiple households requires first that these models are compared to the standard exchange and production general equilibrium models of economic theory. In fact, CGE models are different in several dimensions: (1) There are two kinds of goods (consumption goods and pure production factors, the latter being inputs to the production process without being arguments of consumers' utility functions); (2) Production sets have a somewhat elaborated geometry due to the absence of joint production (of consumption goods), fixed coefficient technologies for consumption goods used as inputs and substitutability between factor inputs; (3) Consumers are endowed only in factors; (4) Existence of governmental and foreign sectors. The operation of these two open sectors creates major differences with the closed general equilibrium models of economic theory. The first thing to do is therefore to close the CGE models of real countries by deleting these two open sectors after having data adjusted for the economic impact of these two sectors. The resulting closed CGE models are then sufficiently close to the standard exchange and production models 
of general equilibrium theory for comparisons to become possible. Nevertheless, the remaining differences suffice to require a fresh study of any question that goes beyond the existence and efficiency of equilibria. In that direction, the special case of two goods, two factors and two consumers has already been considered by Vanek in a paper published in (1963). There, Vanek adheres to the standards of the time by limiting himself to the derivation of formulas expressing the local comparative statics of the equilibria of that model. For example, Vanek does not prove the existence of an equilibrium nor studies the number of equilibria. One goal of the current paper is to take up the theory of closed CGE models where Vanek left it by developing the theory for an arbitrary number of goods, factors and households along the lines of the global approach through the equilibrium manifold and the natural projection, an approach that is particularly fitted for the identification of economies with multiple equilibria as follows from Balasko (1975a, 2011).

That undertaking will be facilitated by the equivalence between the pure HeckscherOhlin model and a suitably defined exchange model for the factors of that HeckscherOhlin model recently proved by Balasko (2013a). The Heckscher-Ohlin and closed CGE models differ only by their production sectors. In the Heckscher-Ohlin model, goods cannot be used as inputs while they can in closed CGE models and, when they are, they contribute to production according to the linear activity model. Our approach will therefore consist in extending the equivalence of Balasko (2013a) to the more general category of production sets considered in the closed CGE models. A few words of explanation about this equivalence between models is in order at this stage. The observation that the demand for consumption goods in both the HeckscherOhlin model and in closed CGE models induces an indirect demand for factors is not new. Rader (1972) and Kehoe (1991) in particular establish properties of the aggregate excess demand function for factors of a given economy viewed as a function of factor prices. As mentioned above, these properties are for a given economy, i.e., for given specifications of the fundamentals of an economy like initial endowments. This approach has enabled Kehoe to extend his earlier work on index numbers and on sufficient conditions for the uniqueness of equilibrium from general equilibrium models with production to CGE models.

The global approach focuses on a collection of economies defined by a set of values of the fundamentals by opposition to a single economy. In the general equilibrium exchange model for example, the fundamentals are represented by the vector $\omega$ defined by the consumers' endowments as in Debreu (1970) and Balasko (1975a). The collection of economies $\Omega$ is also known as the parameter space and is in general the full set of possible endowments. A "model" then consists of a parameter space and of an equilibrium concept. For example, the Edgeworth-Pareto-Bowley box $\Omega$ plus its contract curve and its collection of budget lines is a "model" in the above sense for the two good, two consumer and fixed total resource case. A point $\omega$ in the box $\Omega$ represents the endowment vector for the two consumers. Properties of the economy $\omega$ will deal for example with the number of the equilibria associated with that endowment vector $\omega$. Typical properties of the Edgeworth-Pareto-Bowley box as a "model" are the pathconnectedness of the set of Pareto optima (a.k.a., the contract curve) or the pathconnectedness of the set of (regular) economies that have a unique equilibrium and the inclusion of the contract curve in that set. 
In the exchange model, an equilibrium is a pair $(p, \omega)$ where $\omega \in \Omega$ is the endowment and the price vector $p$ an equilibrium price vector associated with the endowment $\omega$. With $S$ denoting the set of all price vectors $p \in S$, the equilibrium manifold $E$ is then the subset of $S \times \Omega$ that consists of all equilibria. A "model" therefore features its equilibrium manifold $E$ and the "natural projection," the map $\pi: E \rightarrow \Omega$ that is the restriction to the equilibrium manifold of the projection map $S \times \Omega \rightarrow \Omega$. The preimage $\pi^{-1}(\omega)$ is in bijection with the equilibrium price vectors associated with the economy defined by the endowments $\omega \in \Omega$. The natural projection captures among other things all the information about the subsets of $\Omega$ that consist of economies with a unique equilibrium and with multiple equilibria, respectively. An important contribution of the global approach in the general equilibrium model has been to highlight the impact of the quantities of net trade at equilibrium on the number of equilibria: in the exchange model (Balasko 1975a), in general equilibrium models with private ownership of production Balasko (2011) and in the Heckscher-Ohlin model (Balasko 2013a).

Two "models" are equivalent if their natural projections are equivalent smooth mappings in the sense of Differential Topology. In other words, these maps coincide up to some diffeomorphisms of the domain and range. Equivalence is a powerful tool for extending properties related with the number of equilibria from one model, the exchange model in particular, to other general equilibrium models like the model with private ownership of production and the Heckscher-Ohlin model. In the current paper, closed CGE models are shown to be equivalent to suitably defined exchange models for factors. ${ }^{1}$ It follows from that equivalence that all the properties of the exchange model, including those related with the sets of endowments with unique and multiple equilibria, carry over to closed CGE models. That result is new.

Our initial goal was to apply the concept of model equivalence to several closed CGE models with multiple households to identify their sets of economies with multiple equilibria. We also wanted these models to be based on real data. We therefore chose four countries for which CGE models with multiple households and high-quality data were known to exist: USA, Brazil, Spain and Ivory Coast. After having unsuccessfully looked for economies with multiple equilibria, we started to look for reasons that might explain why economies with multiple equilibria were so elusive in those CGE models with multiple households. This led us to focus on the set of equilibrium allocations in the equivalent factor models and to observe, much to our surprise, that these equilibrium factor allocations (or, by equivalence, the factor content of the allocations in consumption goods at equilibrium) were collinear or almost collinear for all consumers in the models of the four countries. This property was all the more remarkable given that the equilibrium allocations in consumption goods in the same models were significantly non-collinear.

Factor proportionality in itself is not new within the setup of the Heckscher-Ohlin model, a model that is rather close to the closed CGE models. For example, factor proportionality has been a staple of international trade theory since Vanek's paper (1968) at least. Nevertheless and to the best of our knowledge, the proportionality of factor contents has not been documented for any CGE model so far. A property that

\footnotetext{
1 This result is the "globalized" version of results of Rader and Kehoe on the aggregate excess demand function for factors of a given economy with goods and factors.
} 
is satisfied by only four countries is a long way from being a universal law. The fact that these four countries were chosen only for their economic differences combined with the quality of the data of their CGE models with multiple households suggests that the property of factor proportionality in those models may nevertheless be more general than one could expect from just these four examples.

This possibility led us to explore the theoretical implications for a CGE model of having the factor content of its equilibrium allocations always proportional for all consumers. That property is extreme and cannot in anyway be considered as resulting from the four examples. The relevance of that property, however, would be strengthened if it is shown to imply other properties that have already been observed in multiple households CGE models. In this paper, we therefore start by showing that, in the simpler exchange model where individual equilibrium allocations are proportional for all possible endowments while preferences are fixed, those preferences are necessarily identical and homothetic. We then show that equilibrium is unique under those circumstances and depends only on the vector of total resources, not on the distribution of these resources among consumers. It then suffices to use the equivalence of the closed CGE model with the exchange model to see that the law of proportional factor contents in multiple households CGE models implies the uniqueness and rigidity of equilibrium prices of goods and factors. The widespread observation that equilibrium price vectors in multiple households CGE models are unique and rigid with respect to endowment redistribution is one more element in favor of the law of proportionality of factor contents.

This paper is organized as follows. The theoretical version of a closed CGE model is defined in Sect. 2. The associated factor exchange model and its equivalence with a closed CGE model is proved in Sect. 3. The equivalence between proportionality of equilibrium allocations and the homotheticity and identity of individual preferences (in exchange economies) is proved in Sect. 4. The proportionality or almost proportionality of factor contents of equilibrium allocations is seen in Sect. 5 on data for the USA, Brazil, Spain and Ivory Coast. Concluding comments end this paper with Sect. 6.

\section{Closed multiple households CGE models}

\subsection{Goods, prices and consumption}

There are two kinds of goods, ordinary consumption goods and pure production factors. Their numbers are denoted by $k$ and $\ell$, respectively. Factors are used only as inputs in production and are substitutable. Consumption goods are arguments of consumers' utility functions. They can also serve in fixed proportion as inputs for the production of other goods. Consumers' endowments consist only of factors.

Prices of consumption good $h$ (with $1 \leq h \leq k$ ) and factor $j$ (with $1 \leq j \leq \ell$ ) are denoted by $q_{h}$ and $p_{j}$, respectively. The $\ell$-th factor is used as numeraire: $p_{\ell}=1$. With $S=\mathbb{R}_{++}^{\ell-1} \times\{1\}$ denoting the set of numeraire normalized factor prices, the Cartesian product $\mathbb{R}_{++}^{k} \times S$ is the price set of the CGE model. 


\section{Utility functions}

There is a finite number $m$ of consumers. Consumer $i$ 's consumption space is the strictly positive orthant $X=\mathbb{R}_{++}^{k}$ of the goods' space. Preferences for goods are represented by a utility function $u_{i}: X \rightarrow \mathbb{R}$ that satisfies the standard assumptions of smooth consumer theory: (1) smoothness; (2) smooth monotonicity, i.e., $D u_{i}\left(x_{i}\right) \in X$ for $x_{i} \in X$; (3) smooth quasi-concavity, i.e., $z=0 \in \mathbb{R}^{k}$ is the only solution to the system $z^{T} D^{2} u_{i}\left(x_{i}\right) z \geq 0$ and $D u_{i}\left(x_{i}\right)^{T} z=0$; (4) the indifference sets $\left\{y_{i} \in X \mid u_{i}\left(y_{i}\right)=\right.$ $\left.u_{i}\left(x_{i}\right)\right\}$ are closed in $\mathbb{R}^{k}$ for $x_{i} \in X$. (For details, see for example Balasko (2011).)

Under these assumptions, consumer $i$ 's demand function is a map $h_{i}: \mathbb{R}_{++}^{k} \times$ $\mathbb{R}_{++} \rightarrow \mathbb{R}_{++}^{k}$ where $x_{i}=h_{i}\left(q, w_{i}\right)$ maximizes the utility $u_{i}\left(x_{i}\right)$ subject to the budget constraint $q \cdot x_{i}=w_{i}$. The demand function $h_{i}$ is homogenous of degree zero: $h_{i}\left(\lambda q, \lambda w_{i}\right)=h_{i}\left(q, w_{i}\right)$ for $\lambda>0$.

Before exchange and production take place, consumer $i$ is endowed with factors represented by the vector $\omega_{i} \in \mathbb{R}_{++}^{\ell}$. We denote by $\omega=\left(\omega_{i}\right)$ the $m$-tuple of endowment vectors and by $\Omega=\left(\mathbb{R}_{++}^{\ell}\right)^{m}$ the endowment set, also known as the parameter space.

\subsection{Production}

Only consumption goods are produced and there is no joint production. Inputs consist of factors and consumption goods. Inputs are denoted negatively, outputs positively.

\section{Consumption goods as inputs}

Consumption goods are used as inputs in fixed proportions. Let $-a_{h^{\prime}}^{h} \leq 0$ denote the quantity of consumption good $h^{\prime} \neq h$ (with $h^{\prime}$ varying from 1 to $k$ ) that is necessary for the (net) production of one unit of good $h$. We define $a_{h}^{h}=0$. These coefficients define a $k \times k$ matrix $A=\left(a_{h^{\prime}}^{h}\right)$ that is non-negative, with zeros on its diagonal. With $I$ denoting the $k \times k$ identity matrix, the input-output matrix of the closed CGE model is by definition the $k \times k$ matrix $I-A$. Following a standard assumption in the input-output literature, this matrix is invertible with a strictly positive inverse. This property expresses the compatibility and feasibility of the various technologies for the production of the consumption goods in the economy.

Invertibility of matrix $I-A$ has a long history. It is Assumption (e) in (1951a, p. 148) or Postulate $C_{1}$ in (1951b, p. 53). This property is equivalent to the HawkinsSimon conditions for matrix I - A (Gale and Nikaido 1965; Hawkins 1948; Hawkins and Simons 1949). Invertibility is satisfied in particular if the input-output matrix $I-A$ is diagonal dominant.

\section{Factors as inputs}

We assume factors to be substitutable when used in the production of consumption goods. It follows from the assumption that (consumption) goods can be used as inputs only in fixed proportion that the quantity of consumption good $h$ that is produced (assuming the availability of sufficient quantities of the other goods for pro- 
duction to take place) is a function $F^{h}\left(\eta_{h}^{1}, \ldots, \eta_{h}^{\ell}\right)$ of the quantities of factor inputs $\left(\eta_{h}^{1}, \ldots, \eta_{h}^{\ell}\right) \in \mathbb{R}_{+}^{\ell}$. In addition, the production function $F^{h}$ for $1 \leq h \leq k$, satisfies: (1) smoothness; (2) necessity of all production factors; (3) monotonicity; (4) homogeneity of degree one (i.e., constant returns to scale); (5) concavity. Typical examples of (production) functions are the Cobb-Douglas and, more generally, the CES production functions.

\section{Production sector's demand for factors}

It is well-known that a unique factor bundle $\eta=g_{h}(p) \in \mathbb{R}_{++}^{\ell}$ minimizes the cost of producing one unit of the consumption good $h$ given the factor price vector $p \in S$. The function $g_{h}: S \rightarrow \mathbb{R}_{++}^{\ell}$ can be thought of as the production sector's demand function for factors to produce one unit of consumption good $h$. Function $g_{h}$ is smooth. The strict inequality $p \cdot g_{h}(p)<p \cdot g_{h}\left(p^{\prime}\right)$ for $p \neq p^{\prime}$ then follows from the definition of $g_{h}(p)$ as minimizing the cost of producing one unit of $\operatorname{good} h$ at price $p \in S$.

\section{The production sector demand matrix}

Let $G(p)$ be the matrix whose $k$ columns are the vectors $g_{h}(p)$ with $1 \leq h \leq k$ :

$$
G(p)=\left[g_{1}(p) g_{2}(p) \ldots g_{k}(p)\right] .
$$

The activity vector that represents the production of one unit of good $h$ in the goods and factor space is the $h$-th column of matrix

$$
\left[\begin{array}{c}
I-A \\
-G(p)
\end{array}\right] .
$$

(Note the minus sign in front of $G(p)$ because inputs are traditionally negative in activity vectors.)

Matrix $B(p)$ defined by

$$
B(p)=G(p)(I-A)^{-1}
$$

will be useful in later developments.

Remark 1 The combination of a Leontieff style input-output technology with factor substitutability is typical of all CGE models. Models with input-output matrix $A=0$ are nothing but standard Heckscher-Ohlin models under factor price equalization. A recent study of those models along the lines of the theory of smooth economies can be found in Balasko (2013a). The first study of a closed CGE model (though without the name) with matrix $A \neq 0$ and in the special case of two goods and two factors is due to Vanek (1963).

\subsection{Equilibrium}

An economy in the closed CGE model is defined by a specific value of the factor endowment vector $\omega=\left(\omega_{i}\right) \in \Omega$. The price vector $(q, p) \in \mathbb{R}_{++}^{k} \times S$ is an equilibrium 
price vector for the economy $\omega \in \Omega$ (of the closed CGE model) if there exists $\gamma \in$ $X=\mathbb{R}_{++}^{k}$ for which the following two equations are satisfied:

$$
\begin{aligned}
\sum_{1 \leq i \leq m} h_{i}\left(q, p \cdot \omega_{i}\right) & =(I-A) \gamma, \\
G(p) \gamma & =\sum_{1 \leq i \leq m} \omega_{i} .
\end{aligned}
$$

Equality (1) simply states the equality between the aggregate demand for goods and the supply of those goods by the production sector given the price vector $(q, p)$. Equality (2) expresses the equality between the demand for factors that are necessary to produce the goods that are to be consumed and the factor supply.

The equilibrium allocation associated with the equilibrium price vector $(q, p) \in$ $\mathbb{R}_{++}^{k} \times S$ is the $m$-tuple $x=\left(x_{i}\right) \in\left(\mathbb{R}_{++}^{k}\right)^{m}$ of consumption goods where $x_{i}=$ $h_{i}\left(q, p \cdot \omega_{i}\right)$ for $1 \leq i \leq m$.

\subsection{Relation between factor prices and goods prices at equilibrium}

Lemma 1 Let $(q, p) \in X \times S$ be an equilibrium price vector. We then have

$$
q=B(p)^{T} p .
$$

Proof The production of each good is a zero-profit operation because of the constant returns to scale. The activities represented by the columns of matrix $\left[\begin{array}{c}I-A \\ -G(p)\end{array}\right]$ have therefore a value equal to zero at the price vector $(q, p)$. This is equivalent to

$$
(I-A)^{T} q-G(p)^{T} p=0 .
$$

Conclusion follows from the invertibility of the input-output matrix $I-A$.

Remark 2 If there is only one pure production factor, Lemma 1 implies that the goods' equilibrium price vector $q$ is constant and independent of the characteristics of the consumption sector, a property known for the Leontieff input-output model as Samuelson's non-substitution theorem (Arrow 1951b).

Remark 3 Lemma 1 tells us that goods' prices are determined by factor prices. The converse of that property, namely the determination of factor prices from the goods prices is the subject of the famous factor price equalization theorem of international trade theory.

\subsection{Factor contents of consumption bundles}

The factor content of the goods bundle $x \in X$ is defined as the factor bundle $y \in \mathbb{R}_{+}^{\ell}$ that minimizes the costs of producing the bundle $x \in X$ given the factor price vector $p \in S$. The factor content of one unit of good $h$ coincides with $g_{h}(p)$. Given the 
absence of joint production, the factor content of the goods bundle $x \in X$ is then equal to

$$
x^{1} g_{1}(p)+\cdots+x^{k} g_{k}(p)=G(p) x,
$$

where $x$ stands for a column matrix in the expression $G(p) x$.

Note that the factor content of the goods bundle $x$ vary with the factor price vector $p \in S$.

\section{Factor exchange models associated with closed CGE models}

The construction of the factor exchange model associated with a closed CGE model is adapted from Balasko (2013a). Account has to be made for consumption goods being also used as production inputs following the linear activity model. Proofs of the equivalence of the two models have to be adapted because of this difference.

The "goods" in the factor exchange model are the $\ell$ factors in the original CGE model. With each consumer $i$ of the closed CGE model is associated a consumer denoted by the same letter $i$. That consumer $i$ has the same endowment vector $\omega_{i} \in$ $\mathbb{R}_{++}^{\ell}$ as the eponymous consumer in the closed CGE model. We now define this consumer's utility function for factors.

\subsection{Utility functions for factors}

With $\hat{u}_{i}\left(q, w_{i}\right)$ denoting the indirect utility $\hat{u}_{i}\left(q, w_{i}\right)=u_{i}\left(h_{i}\left(q, w_{i}\right)\right)$, the indirect utility function for factors is defined for $\left(p, w_{i}\right) \in S \times \mathbb{R}_{++}$by $\hat{v}_{i}\left(p, w_{i}\right)=$ $\hat{u}_{i}\left(B(p)^{T} p, w_{i}\right)$.

The direct utility $v_{i}\left(y_{i}\right)$ of $y_{i} \in \mathbb{R}_{++}^{\ell}$ is then defined by the formula $v_{i}\left(y_{i}\right)=$ $\inf _{p \in S} \hat{v}_{i}\left(p, p \cdot y_{i}\right)$ that relates direct and indirect utility functions in general.

Proposition 1 The utility function for factors $v_{i}: \mathbb{R}_{++}^{\ell} \rightarrow \mathbb{R}$ satisfies the properties (i) to (iv) of Sect 2.1.

Proof It suffices to reproduce the proof given in Balasko (2013b).

\subsection{Demand functions for factors}

In this part, factor prices are not normalized (to be able to take derivatives with respect to the price $p_{\ell}$ of the numeraire in the formulation of Roy's identity). Let $f_{i}: \mathbb{R}_{++}^{\ell} \times \mathbb{R}_{++} \rightarrow \mathbb{R}_{++}^{\ell}$ denote consumer $i$ 's demand function for factors. By definition, $f_{i}\left(p, w_{i}\right)$ maximizes $v_{i}\left(y_{i}\right)$ subject to the budget constraint $p \cdot y_{i}=w_{i}$. We then have:

Proposition $2 f_{i}\left(p, w_{i}\right)=B(p) h_{i}\left(B(p)^{T} p, w_{i}\right)$.

Step 1: $\partial_{p}\left(B(p)^{T} p\right)=B(p)^{T}$. The derivative of the matrix product $B(p)^{T} p$ is equal to

$$
\partial_{p}\left(B(p)^{T} p\right)=B(p)^{T}+\left(\partial_{p} B(p)\right)^{T} p .
$$


From $B(p)=G(p)(I-A)^{-1}$, it comes $B(p)^{T}=\left((I-A)^{T}\right)^{-1} G(p)^{T}$ and, therefore $\partial_{p} B(p)^{T}=\left((I-A)^{T}\right)^{-1}\left(\partial_{p} G(p)\right)^{T}$. The homogeneity of degree zero of each function $g_{j}(p)$ implies

$$
\left(\partial_{p} G(p)\right)^{T} p=0
$$

From these equalities follows $\left.\left(\partial_{p} B(p)\right)^{T} p=\left((I-A)^{T}\right)^{-1}\right)^{T}\left(\partial_{p} G(p)\right)^{T} p=0$ and, therefore, $\partial_{p}\left(B(p)^{T} p\right)=B(p)^{T}$.

Step 2: Computations of $\partial_{w_{i}} \hat{v}_{i}\left(p, w_{i}\right)$ and $\partial_{p} \hat{v}_{i}\left(p, w_{i}\right)$. Let $q=B(p)^{T} p$. From $\hat{v}_{i}\left(p, w_{i}\right)=\hat{u}_{i}\left(B(p)^{T} p, w_{i}\right)$, it comes

$$
\partial_{w_{i}} \hat{v}_{i}\left(p, w_{i}\right)=\partial_{w_{i}} \hat{u}_{i}\left(B(p)^{T} p, w_{i}\right)=\partial_{w_{i}} \hat{u}_{i}\left(q, w_{i}\right)
$$

The chain rule yields

$$
\partial_{p} \hat{v}_{i}\left(p, w_{i}\right)^{T}=\partial_{q} \hat{u}_{i}\left(q, w_{i}\right)^{T} \partial_{p}\left(B(p)^{T} p\right)
$$

After transposition, it follows from Step 1 that

$$
\partial_{p} \hat{v}_{i}\left(p, w_{i}\right)=B(p) \partial_{q} \hat{u}_{i}\left(q, w_{i}\right) .
$$

Step 3: The function $\left(p, w_{i}\right) \rightarrow B(p) h_{i}\left(B(p)^{T} p, w_{i}\right)$ satisfies Roy's identity for $\hat{v}_{i}$. Let us write Roy's identity for the indirect utility function $\hat{u}_{i}\left(q, w_{i}\right)$ and its associated demand function $h_{i}\left(q, w_{i}\right)$ :

$$
\partial_{w_{i}} \hat{u}_{i}\left(q, w_{i}\right) h_{i}\left(q, w_{i}\right)=-\partial_{q} \hat{u}_{i}\left(q, w_{i}\right) \text {. }
$$

Left multiplication by $B(p)$ yields

$$
\partial_{w_{i}} \hat{u}_{i}\left(q, w_{i}\right) B(p) h_{i}\left(q, w_{i}\right)=-B(p) \partial_{q} \hat{u}_{i}\left(q, w_{i}\right) .
$$

Using Step 2, this equality can be rewritten as

$$
\partial_{w_{i}} \hat{v}_{i}\left(p, w_{i}\right) B(p) h_{i}\left(B(p)^{T} p, w_{i}\right)=-\partial_{p} \hat{v}_{i}\left(p, w_{i}\right) .
$$

It then follows from Roy's identity

$$
\partial_{w_{i}} \hat{v}_{i}\left(p, w_{i}\right) f_{i}\left(p, w_{i}\right)=-\partial_{p} \hat{v}_{i}\left(p, w_{i}\right)
$$

satisfied by the demand function $f_{i}\left(p, w_{i}\right)$ associated with the indirect utility $\hat{v}_{i}$ that $f_{i}\left(p, w_{i}\right)=B(p) h_{i}\left(B(p)^{T} p, w_{i}\right)$. 


\subsection{Equilibrium of the factor exchange model}

The factor exchange economy is the exchange economy with commodity space the actual factor space $\mathbb{R}^{\ell}$, with consumer $i$ 's endowments represented by the vector $\omega_{i} \in \mathbb{R}_{++}^{\ell}$ and utility function $v_{i}: \mathbb{R}_{++}^{\ell} \rightarrow \mathbb{R}$ for $i$ varying from 1 to $m$.

The price vector $p \in S$ is an equilibrium price vector of the factor exchange economy defined by the endowment vector $\omega=\left(\omega_{i}\right)$ if

$$
\sum_{1 \leq i \leq m} f_{i}\left(p, p \cdot \omega_{i}\right)=\sum_{i} \omega_{i}
$$

The equilibrium allocation associated with the equilibrium price vector $p \in S$ is the $m$-tuple $x=\left(x_{i}\right)$ where $x_{i}=f_{i}\left(p, p \cdot \omega_{i}\right)$ for $1 \leq i \leq m$.

\section{Equivalence of the closed CGE model and the associated factor exchange model}

The closed CGE economy is defined by the endowment vector $\omega=\left(\omega_{i}\right)$ (with preferences for goods represented by utility functions $u_{i}$ and production technologies described in Sect. 2.2). The exchange economy is defined by the same endowment vector (and preferences for factors now defined by the utility functions $v_{i}$ ). These two economies are equivalent in the following sense:

Proposition 3 The price vector $(q, p) \in X \times S$ is an equilibrium price vector of the closed CGE economy if and only if the price vector $p \in S$ is an equilibrium price vector of the exchange economy and $q=B(p)^{T} p$.

Proof The price vector $(q, p) \in \mathbb{R}_{++}^{k} \times S$ is an equilibrium price vector of the closed CGE economy if and only if

$$
\begin{aligned}
\sum_{1 \leq i \leq m} h_{i}\left(q, p \cdot \omega_{i}\right) & =(I-A) \gamma, \\
G(p) \gamma & =\sum_{1 \leq i \leq m} \omega_{i} .
\end{aligned}
$$

Left multiplication by $G(p)(I-A)^{-1}$ of (7) combined with (8) yields

$$
\sum_{1 \leq i \leq m} B(p) h_{i}\left(q, p \cdot \omega_{i}\right)=\sum_{1 \leq i \leq m} \omega_{i}
$$

It follows from Proposition 2 that the price vector $p \in S$ satisfies the equilibrium equation of the exchange economy while the equality $q=B(p)^{T} p$ is Lemma 1 .

Conversely, let $p \in S$ be an equilibrium price vector of the exchange economy. Let $q=B(p)^{T} p \in X$ and $\gamma \in \mathbb{R}^{k}$ be defined by 


$$
\gamma=(I-A)^{-1} \sum_{1 \leq i \leq m} h_{i}\left(q, p \cdot \omega_{i}\right) .
$$

Each $h_{i}\left(q, p \cdot \omega_{i}\right)$ belongs to the strictly positive orthant $X$ of $\mathbb{R}^{k}$. So does the sum $\sum_{1 \leq i \leq m} h_{i}\left(q, p \cdot \omega_{i}\right)$. It then suffices to observe that the matrix $I-A$ being invertible with a strictly positive inverse, the column matrix $\gamma$ is the matrix product of strictly positive matrices and is therefore strictly positive. This implies $\gamma \in X$. Left multiplication by $G(q)$ of equality (10) gives us

$$
G(q) \gamma=B(q) \sum_{1 \leq i \leq m} h_{i}\left(q, p \cdot \omega_{i}\right)
$$

With $p \in S$ an equilibrium price vector of the factor exchange economy, the above right-hand side term is equal to $\sum_{1 \leq i \leq m} \omega_{i}$, from which follows the equality

$$
G(q) \gamma=\sum_{1 \leq i \leq m} \omega_{i}
$$

which is Eq. (2).

4.1 Factor contents of equilibrium allocations of the closed CGE economy

Let $(q, p) \in X \times S$ be an equilibrium price vector of the closed CGE economy. The equilibrium allocation $y=\left(y_{i}\right) \in\left(\mathbb{R}_{++}^{\ell}\right)^{m}$ associated with the equilibrium price vector $p \in S$ for the factor exchange economy is related to the equilibrium allocation $x=\left(x_{i}\right)$ of the closed CGE economy by:

Proposition 4 For $1 \leq i \leq m$, consumer $i$ 's factor demand $y_{i}=f_{i}\left(p, p \cdot \omega_{i}\right)$ at the equilibrium price vector $p \in S$ is the factor content of consumer $i$ 's goods demand $h_{i}\left(q, p \cdot \omega_{i}\right)$ for the equilibrium price vector $(q, p) \in X \times S$.

Proof The proposition is equivalent to the equality $f_{i}\left(p, p \cdot \omega_{i}\right)=B(p) h_{i}\left(q, p \cdot \omega_{i}\right)$. This follows from Proposition 2 combined with Proposition 3.

\subsection{Properties of the closed CGE model}

The properties of any closed CGE model can now be derived from those of its equivalent exchange model. When dealing with models based on real data, we are therefore going to compute and compare the factor contents of equilibrium allocations in the closed CGE model with the factor endowments of the consumers since many properties of the equivalent factor exchange model depend on that net trade vector in factor contents. 


\section{Proportionality of factor contents: the theory}

The real data that will be considered in the next section will reveal the baffling property that the factor contents of equilibrium allocations are proportional for the closed CGE models of four countries. In this section, we analyze the theoretical implications of that proportionality property that is assumed to hold for any vector of total resources.

Let $r \in \mathbb{R}_{++}^{\ell}$ be the vector of total resources in an exchange model with $\ell$ goods and $m$ consumers: $r=\omega_{1}+\cdots+\omega_{m}$. Let $P(r)$ denote the set of Pareto optima associated with those total resources. Let $\Sigma=\left\{\lambda=\left(\lambda_{1}, \ldots, \lambda_{m}\right) \in \mathbb{R}_{++}^{m} \mid \sum_{i} \lambda_{i}=1\right\}$ be the strictly positive $m$-simplex.

Proposition 5 The following two properties are equivalent:

(i) $P(r)=\left\{\left(\lambda_{1} r, \ldots, \lambda_{m} r\right) \mid \lambda \in \Sigma\right\}$ for all $r \in \mathbb{R}_{++}^{\ell}$.

(ii) Consumers have identical homothetic preferences.

Proof $(i) \Longrightarrow$ (ii) Let $x_{1}=f_{1}\left(p, w_{1}\right)$ with $\left(p, w_{1}\right) \in S \times \mathbb{R}_{++}$. Let $x_{2}, \ldots, x_{m}$ such that $x_{2}=\cdots=x_{m}=x_{1}=\bar{x} \in \mathbb{R}_{++}^{\ell}$. With $r=x_{1}+\cdots+x_{m}=m \bar{x}$, it follows from (i) that the allocation of goods defined by the $m$-tuple $x=\left(x_{1}, \ldots, x_{m}\right)$ belongs to $P(m \bar{x})$ and is a Pareto optimum. As such, it is supported by some price vector that also supports the individual allocations, hence the commodity bundle $x_{1}$ for consumer 1. This implies that the price vector $p$ supports all individual allocations $x_{2}, \ldots, x_{m}$ in addition to $x_{1}$. It also comes $w_{i}=p \cdot x_{i}=p \cdot x_{1}$ for $i \geq 2$. As a consequence, it comes $f_{i}\left(p, w_{i}\right)=x_{i}=x_{1}=f_{1}\left(p, w_{1}\right)$, which proves the equality of demand functions $f_{1}=\cdots=f_{m}$.

Let $x_{1}=\bar{x}$ be arbitrary and $x_{2}=\lambda \bar{x}$, for some $\lambda>0$. Define $x_{3}=\cdots=x_{m}=\bar{x}$. It follows from $(i)$ that $x=\left(x_{1}, x_{2}, \ldots, x_{m}\right)$ that belongs to $P(m \bar{x})$ is Pareto optimal. Each allocation $x_{i}$ is supported by the same price vector $p \in S$, which applies to $x_{1}$ and to $\lambda x_{1}$ for any $\lambda>0$. This proves the relation $f_{1}\left(p, \lambda w_{1}\right)=\lambda f_{1}\left(p, w_{1}\right)$. This property of the demand function $f_{1}$ is equivalent to the homotheticity of consumer 1 's preferences.

$(i i) \Longrightarrow(i)$. Let $p$ be the supporting price vector of the Pareto optimum $x=$ $\left(x_{1}, \ldots, x_{m}\right)$. The price vector $p$ supports the individual allocation $x_{2}, \ldots, x_{m}$ for consumer $2, \ldots, m$ respectively. Their preferences being identical to those of consumer 1 , the goods bundles $x_{2}, \ldots, x_{m}$ are also supported by the same price vector $p$ but now for consumer 1's preferences. Homotheticity of consumer 1's preferences then implies that these allocations are all collinear.

Proposition 6 Let all consumers have identical homothetic preferences.

(i) Equilibrium is unique for all endowment vectors $\omega=\left(\omega_{i}\right) \in \mathbb{R}_{++}^{\ell}$.

(ii) The unique equilibrium price vector $p$ associated with the endowment vector $\omega=\left(\omega_{i}\right)$ depends only on the total resources $r=\sum_{i} \omega_{i}$, not on the distribution of resources between the consumers.

Proof This is a direct consequence of Gorman's well-known aggregation theorem (Gorman 1953). Here is an alternative simple proof. We need to prove only (ii) since (i) follows from (ii). 
Let $p \in S$ be the price vector that supports the factor bundle $r \in \mathbb{R}_{++}^{\ell}$ for anyone of the $m$ (identical) consumers. This means that $r=f_{i}(p, p \cdot r)$ for $1 \leq i \leq m$. Let $x=\left(x_{i}\right)$ be a Pareto optimum associated with those total resources $r \in \mathbb{R}_{++}^{\ell}$. It follows from Proposition 5 that there exists $\lambda=\left(\lambda_{i}\right) \in \Sigma_{m}$ in the open m-simplex such that $x_{i}=\lambda_{i} r=\lambda_{i} f_{i}(p, p \cdot r)$. By the homotheticity of preferences, it comes $x_{i}=f_{i}\left(p, p \cdot \lambda_{i} r\right)=f_{i}\left(p, p \cdot x_{i}\right)$ for $1 \leq i \leq m$. This proves that the price vector $p$ supports all the Pareto optimum allocations that belong to $P(r)$, which proves (ii).

\section{Applications}

The original SAMs at the origin of the data used in this paper have been compiled for the USA (1980) by Adelman and Robinson (1986), for Brazil (2003) by Tourinho et al. (2006), for Spain (1980) by Kehoe et al. (1988) and for Ivory Coast (1986) by Thornbecke (2000).

The equilibrium allocations and factor contents for the closed CGE models are taken from Tourinho (2013). This document can be downloaded under the name of "Computation of SAMs for closed CGE models" at "www.researchgate.net" after having searched for the publications of Octavio Tourinho. Two additional documents "SAMs aggregation" and "Simple SAMs" available at the same site and by the same author provide the spreadsheets used to compute the data in this paper.

In these four examples, the angles (measured in degrees) made by the vectors of factor contents are very small. The approximate collinearity of the vectors of factor contents is to be contrasted with the angles that are significantly different from zero observed between most bundles of consumption goods (with the exception of the USA).

\section{USA}

Angles of goods bundles and of factor contents respectively are quite small. Nevertheless, the angles made by the factor contents are consistently one-tenth of the angles of the goods bundles (Table 1).

\section{Brazil}

Angles of goods bundles are quite large, at variance with those computed for the USA. This can easily be justified by the diversity of consumption patterns in Brazil. Note that

Table 1 USA: angles for goods bundles and factor contents

\begin{tabular}{|c|c|c|c|c|c|c|c|}
\hline \multicolumn{2}{|c|}{ Household income class } & \multicolumn{3}{|c|}{ Goods bundles } & \multicolumn{3}{|c|}{ Factor contents } \\
\hline & & $\mathrm{C}$ & B & A & $\mathrm{C}$ & B & A \\
\hline Low $40 \%$ & $\mathrm{C}$ & 0 & 2.4 & 3.4 & 0 & 0.2 & 0.3 \\
\hline Medium $40 \%$ & B & 2.4 & 0 & 1.0 & 0.2 & 0 & 0.1 \\
\hline Upper $20 \%$ & A & 3.4 & 1.0 & 0 & 0.3 & 0.1 & 0 \\
\hline
\end{tabular}


Table 2 Brazil: angles for goods bundles and factor contents

\begin{tabular}{|c|c|c|c|c|c|c|c|c|c|}
\hline \multirow[t]{2}{*}{ Household income class } & & \multicolumn{4}{|c|}{ Goods bundles } & \multicolumn{4}{|c|}{ Factor contents } \\
\hline & & $\mathrm{D}$ & $\mathrm{C}$ & B & A & $\mathrm{D}$ & $\mathrm{C}$ & B & A \\
\hline Income $\leq 3$ S.M. & $\mathrm{D}$ & 0 & 12 & 32 & 57 & 0 & 1.0 & 0.5 & 2.6 \\
\hline 3 S.M. $<$ income $\leq 10$ S.M. & $\mathrm{C}$ & 12 & 0 & 23 & 50 & 1.0 & 0 & 1.0 & 0.6 \\
\hline 10 S.M. $<$ income $\leq 30$ S.M & $\mathrm{B}$ & 32 & 23 & 0 & 29 & 0.5 & 1.0 & 0 & 2.6 \\
\hline Income > 30 S.M. & A & 57 & 50 & 29 & 0 & 2.6 & 3.6 & 2.6 & 0 \\
\hline
\end{tabular}

Table 3 Spain: angles for goods bundles and factor contents

\begin{tabular}{|c|c|c|c|c|c|c|c|c|c|}
\hline \multirow[t]{2}{*}{ Household income class } & & \multicolumn{4}{|c|}{ Goods bundles } & \multicolumn{4}{|c|}{ Factor contents } \\
\hline & & RQ & RNQ & PQ & PNQ & RQ & RNQ & PQ & PNQ \\
\hline Rich and qualified & RQ & 0 & 4.8 & 5.1 & 10.5 & 0 & 0.4 & 0.5 & 0.8 \\
\hline Rich and non-qualified & RNQ & 4.8 & 0 & 1.9 & 6.1 & 0.4 & 0 & 0.2 & 0.4 \\
\hline Poor and qualified & PQ & 5.1 & 1.9 & 0 & 6.7 & 0.5 & 0.2 & 0 & 0.2 \\
\hline Poor and non-qualified & PNQ & 10.5 & 6.1 & 6.7 & 0 & 0.8 & 0.4 & 0.2 & 0 \\
\hline
\end{tabular}

Table 4 Ivory Coast: angles for goods bundles

\begin{tabular}{llcccccc}
\hline Household income class & \multicolumn{9}{l}{ Goods bundles } & & \\
\cline { 3 - 7 } & & RW & RLS & RLL & UL & UH & CAP \\
\hline Rural workers & RW & 0 & 6 & 12 & 7 & 22 & 73 \\
Rural landowners (small) & RLS & 6 & 0 & 8 & 6 & 17 & 70 \\
Rural landowners (large) & RLL & 12 & 8 & 0 & 7 & 11 & 70 \\
Urban low education & UL & 7 & 6 & 7 & 0 & 18 & 74 \\
Urban high education & UH & 22 & 17 & 11 & 18 & 0 & 62 \\
Capitalists & CAP & 73 & 70 & 70 & 74 & 62 & 0 \\
\hline
\end{tabular}

S.M. stands for the 2003 Brazilian minimum wage. As for the USA, angles between factor contents are about one-tenth of those between goods bundles (Table 2).

\section{Spain}

Angles between goods bundles lie in between the USA and Brazil. Here, again, angles of factor contents are approximately one-tenth of those for goods bundles (Table 3).

\section{Ivory Coast}

The data do not exclude a possible though minor deviation from proportionality of factor contents Tables $(4,5)$. 
Table 5 Ivory Coast: angles for factor contents

\begin{tabular}{llcccccc}
\hline Household income class & \multicolumn{7}{l}{ Factor contents } \\
\cline { 3 - 8 } & & RW & RLS & RLL & UL & UH & CAP \\
\hline Rural workers & RW & 0 & 2.4 & 5.4 & 2.7 & 9.0 & 16.8 \\
Rural landowners (small) & RLS & 2.4 & 0 & 3.0 & 0.6 & 6.7 & 14.6 \\
Rural landowners (large) & RLL & 5.4 & 3.0 & 0 & 2.8 & 3.7 & 12.1 \\
Urban low education & UL & 2.7 & 0.6 & 2.8 & 0 & 6.5 & 14.7 \\
Urban high education & UH & 9.0 & 6.7 & 3.7 & 6.5 & 0 & 8.9 \\
Capitalists & CAP & 16.8 & 14.6 & 12.1 & 14.7 & 8.9 & 0 \\
\hline
\end{tabular}

\section{Conclusion}

To the best of our knowledge, there are no explicit references to the proportionality of factor contents at equilibrium in the literature on CGE models. That property is very intriguing. The fact that it is satisfied by the SAMs of four countries does not suffice to turn it into a general law. One reason is that it is difficult to believe that all real consumers have implicit preferences for factor contents that are identical and homothetic. Here, we are not dealing with real consumers or households but with highly aggregated representations of those consumers for the sake of CGE modeling. The goal of these CGE models is to produce numerical models that can account for general equilibrium effects that are beyond the reach of partial equilibrium analysis. This goal is very ambitious because many of these effects involve multiple equilibria and multiple households. CGE models with multiple households therefore carry the promise of multiple equilibria and, even more importantly, of non-linearities in the relations between economies and their equilibria. This hope is dampened for the CGE models with multiple households that satisfy the law of proportionality of factor contents. The different households then have the same homothetic preferences for factors, preferences that satisfy the Gorman and Nataf perfect conditions for aggregation (Gorman 1953; Nataf 1953). Despite featuring multiple households, these CGE models behave like CGE models with a unique or representative household. The different households are not sufficiently diverse to allow for multiple equilibria.

The proportionality of factor contents is likely to be an artifact of current CGE modeling. Further research is necessary if the goal is to find ways of aggregating household preferences without wiping away the diversity and non-linearities that underlie general equilibrium effects.

\section{References}

Adelman, I., Robinson, S.: US agriculture in a general equilibrium framework: analyis with a social accounting matrix. Am. J. Agric. Econ. 68, 1196-1207 (1986)

Arrow, K.: Alternative proof of the substitution theorem for Leontieff models in the general case. In: Koopmans, T. (ed.) Activity Analysis of Production and Allocation, pp. 155-164. Wiley, New York (1951) 
Arrow, K.: An extension of the basic theorems of classical welfare economics. In: Neyman, J. (ed.) Proceedings of the Second Berkeley Symposium on Mathematical Statistics and Probability, pp. 507-532. University of Berkeley Press, Berkeley (1951)

Arrow, K., Debreu, G.: Existence of an equilibrium for a competitive economy. Econometrica 22, 265-290 (1954)

Arrow, K., Hahn, F.: General Competitive Analysis. Holden-Day, San Francisco (1971)

Balasko, Y.: Some results on uniqueness and on stability of equilibrium in general equilibrium theory. J. Math. Econ. 2, 95-118 (1975)

Balasko, Y.: General Equilibrium Theory of Value. Princeton University Press, Princeton, NJ (2011)

Balasko, Y.: Heckscher-Ohlin explained by Walras. Working paper (2013)

Balasko, Y.: The impact of market segmentation on the stability of competitive equilibria. In preparation (2013)

Debreu, G.: Economies with a finite set of equilibria. Econometrica 38, 387-392 (1970)

Dierker, E.: Two remarks on the number of equilibria of an economy. Econometrica 40, 951-953 (1972)

Gale, D., Nikaido, H.: The Jacobian matrix and the global univalence of mappings. Math. Ann. 159, 81-93 (1965)

Gorman, W.: Community preference fields. Econometrica 21, 63-80 (1953)

Hawkins, D.: Some conditions of macroeconomic stability. Econometrica 16, 309-322 (1948)

Hawkins, D., Simons, H.: Note: some conditions of macroeconomic stability. Econometrica 17, 245-248 (1949)

Kehoe, T.J.: An index theorem for general equilibrium models with production. Econometrica 48, 12111232 (1980)

Kehoe, T.J.: Regular production economies. J. Math. Econ. 10, 147-176 (1982)

Kehoe, T.J.: Computing all of the equilibria of economies with two factors of production. J. Math. Econ. 13, 207-223 (1984)

Kehoe, T.J.: Mulplicity of equilibria and comparative statics. Q. J. Econ. 100, 119-147 (1985)

Kehoe, T.J.: Computation and multiplicity of equilibria. In: Hildenbrand, W., Sonnenschein, H. (eds.) Handbook of Mathematical Economics, vol. IV, pp. 2051-2144. North-Holland, Amsterdam (1991)

Kehoe, T.J., Manresa, A., Polo, C., Sancho, F.: Una matriz de contabilidad social de la economia espa nola (version revisada). Estatistica Espa nola 30, 5-33 (1988)

Kehoe, T.J., Whalley, J.: Uniqueness of equilibrium in large-scale numerical general equilibrium models. J. Public Econ. 28, 247-254 (1985)

Koopmans, T.: Alternative proof of the substitution theorem for Leontief models in the case of three industries. In: Koopmans, T. (ed.) Activity Analysis of Production and Allocation, pp. 147-154. Wiley, New York (1951)

Koopmans, T.: Analysis of production as an efficient combination of activities. In: Koopmans, T. (ed.) Activity Analysis of Production and Allocation, pp. 33-97. Wiley, New York (1951)

McKenzie, L.: On equilibrium in Graham's model of world trade and other competitive systems. Econometrica 22, 147-161 (1954)

Nataf, A.: Sur des questions d'ÇÖagrégation en économétrie. Publications de l'Institut de Statistique de l'Université de Paris, 4:5-Çô-61 (1953)

Negishi, T.: The stability of a competitive economy: A survey article. Econometrica 30, 635-669 (1962)

Rader, T.: General equilibrium theory with complementary factors. J Econ Theory 4, 372-380 (1972)

Thornbecke, E.: The use of social accounting matrices in modeling (revised version). In: 26th General Conference of the International Association for Research in Income and Wealth. Cracow Poland (2000)

Tourinho, O.: Obtaining simple SAMs from full SAMs: four examples, p. 16. Working paper (2013)

Tourinho, O., da Silva, N., Alves, Y.: Uma matriz de contabilidade social (SAM) para o Brasil. Technical report 2141, IPEA, Rio de Janeiro (2006)

Vanek, J.: Variable factor proportions and interindustry flows in the theory of international trade. Q. J. Econ. 77, 129-142 (1963)

Vanek, J.: The factor proportions theory: the $n$ factor case. Kyklos 21, 749-756 (1968)

Wald, A.: On some systems of equations of mathematical economics. Econometrica 19:368-403, 1951. English translation of "Über einige Gleichungssysteme der Mathematischen Ökonomie". Zeitschrift für Nationalökonomie 7:637-670 (1936)

Walras, L.: Eléments d'Economie Politique Pure, 1st edn. Corbaz, Lausanne (1874) 